\title{
CALCUL ÉLÉMENTAIRE DES VALEURS DES CHALEURS SPÉCIFIQUES \\ D'UN LIQUIDE ET DE SA VAPEUR SATURÉE A LA TEMPÉRATURE CRITIQUE;
}

\author{
P.U M. H IIUNOHY.
}

La chaleur spécifique d'un liquide au contact de sa vapeur et la chaleur spécifique de la vapeur saturée éprouvent, quand la température seèlève, des variations bien connues depuis les expériences de M. Mathias et les recherches théoriques de M.M. I) uh.'met liaveau. On sait en particulier que les valeurs de ces chaleurs spécifiques tendent respectivement vers $+\infty$ et vers $-\infty$, si la température croit jusqu'au point critique.

On peut s'en rendre compte aisément par un raisonnement ćlémentaire; il suffit d'observer que, si la température s'élève, le volume d'une masse liquide augmente, tandis qu'au contraire on doit diminuer le volume d'une masse de vapeur pour la maintenir à la tension maxima. En d'autres termes, si l'on désigne par $u$ et $u^{\prime}$ les volumes spécifiques d'un liquide et de sa vapeur, à la température $t$ et suus la tension maxima correspondante $p$, on a :

$$
\frac{d u}{d t}>n, \quad \frac{d u i}{d t}=0 .
$$

On peut d'ailleurs écrire:

$$
\frac{d u}{d t}=\frac{d u}{d p} \frac{d p}{d t} . \quad \frac{d u}{d t} \quad d \|^{\prime} \frac{d p}{d t}
$$

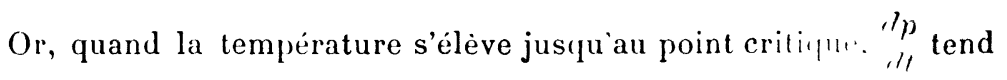
vers une valeur finie; au contraire, les valeurs absolues "! "., "t de $\frac{d u^{\prime}}{d l^{\prime}}$ tendent vers l'infini, puisque la tangente au print ritique a l'isotherme correspondante est parallèle à l'axe des volumun.

On a donc à la température critique :

$$
\frac{d u}{d t}=+\infty, \quad \text { (2) } \quad \frac{d u^{\prime}}{d t}=-\infty .
$$

Supposons maintenant qu'on élive la température de l'unit. de m..... d. la vapeur de $t$ it $t+d t$, en purtant sa pression à la muselle tension maxima $p+d p$. Son volume variera de la quintit. néga- 
tive $d u^{\prime}$, et il fauıra lui fournir la quantité de chaleur $d Q^{\prime}$. Soit $m^{\prime}$ la chaleur spécifique de la vapeur saturée, à la température $t$; on a :

$$
d Q^{\prime}=n^{\prime} d t
$$

On'sait d'ailleurs que l'on a également :

$$
d Q^{\prime}=c^{\prime} d t+l^{\prime} d u^{\prime},
$$

$c^{\prime}$ désignant la chaleur spécifique à volume constant, et $l^{\prime}$ la chaleur latente de dilatation à la température $t$ de la vapeur sèche. On peut supposer en effet que la vapeur est d'abord chauffée à volume constant de $t$ à $t+d t$, puis comprimée à température constante jusqu'à la pression $p+d p$, et, pendant ces deux opérations, elle n'est pas saturante.

Pendant la compression à température constante, il se produit à la fois un travail interne qu'on peut représenter par la quantité de chaleur $l_{1}^{\prime} d u^{\prime}$ ( $l_{1}^{\prime}$ désignant la chaleur latente interne de dilatation), et un travail externe qui a pour expression $p d u^{\prime}$. On a ainsi :

$$
l^{\prime} d u^{\prime}=\left(l_{1}^{\prime}+\frac{p}{\mathrm{E}}\right) d u^{\prime} .
$$

L'expression de $d \mathrm{Q}^{\prime}$ devient finalement :

$$
d Q^{\prime}=m^{\prime} d t=c^{\prime} d t+\left(l_{1}^{\prime}+\frac{p}{\mathrm{E}}\right) d u^{\prime}
$$

et en divisant par $d t$ :

$$
m^{\prime}=c^{\prime}+\left(l_{1}^{\prime}+\frac{p}{\mathrm{E}}\right) \frac{d u^{\prime}}{d t}
$$

Si l'on désigne par $c$ la chaleur spécifique à volume constant, par $l_{1}$ la chaleur latente interne de dilatation du liquide à la température $t$, on aurait de même pour expression de la chaleur spécifique $m$ du liquide au contact de sa vapeur, à la température $t$ :

$$
m=c+\left(l_{1}+\frac{p}{\mathrm{E}}\right) \frac{d u}{d t}
$$

Considérons la dernière relation. Toutes les quantités qui figurent dans le second membre sont positives, et à la température critique :

$$
\frac{d u}{d t}=+\infty
$$




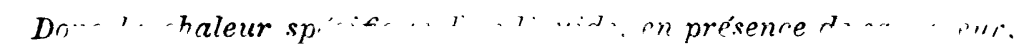
est $c$ ient posit

rature siéleve jusquiau point critique.

Considérons enfin le second membre de la relation (3. Le premier terme, $r^{\prime}$, est positif, ainsi que le premier facteur du deuxième terme; mais le dernier facteur $\frac{d u^{\prime}}{d t}$ est négatif et devient égal à $-x$ à la température critique.

Donc la chaleur spécifique de la vapeur satil, j, j, it, ou positive tant que la température est il....

mais elle est nécessairement "f'gutire it quand la température devient de plus en plus voistne de lı,".,", critique.

Chaleur spécifique de la vapeur d'eau saturée.

La chaleur spécifique de la vapeur d'eau saturée est-clle toujours négative, ou présente-t-elle deux points d'inversion, comme celle de l'anhydride sulfureux?

On sait que la relation bien connue

$$
m^{\prime}=m+\frac{d \mathrm{~L}}{d \mathrm{~T}}-\frac{\mathrm{L}}{\mathrm{T}}{ }^{(1)}
$$

permet de calculer la chaleur spécifique d'une vapeur saturée quand on connaît la chaleur totale et la chaleur latente de vaporisation. Les valeurs de ces deux quantités ont été déterminées par Regnault jusqu’à la température de $190^{\circ}$ (468 ${ }^{\circ}$ absolus). En tenant compte des résultats classiques des expériences de Regnault, la relation précédente devient :

$$
m^{\prime}=-\frac{800,8}{\mathrm{~T}}+1,056-0,0000225 \mathrm{~T} \mathrm{~T}+0,0000003 \mathrm{~T}^{2}
$$

Si on regarde cette dernière équation comme exact. is

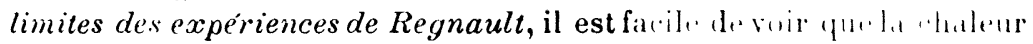
spécifique de la vapeur d'eau saturée reste constamment négative.

(') On peut remalifuer quà la températur. critique le second membre de cette relatun prend la forme $(x-\infty$. Il ne peut donc pas faire connaitre, la valeur de $m$ au point critique. 
Traçons (fig. 1) la branche AB de la courbe reprisentée par cette équation, entre les limites précitées, les coordonnées du point extrême $\mathrm{B}$ étant $\mathrm{T}=468^{\circ}$ et $m=-0,69 \ddot{\text { s. et menons la tangente à }}$ la courbe au point $B$. On peut connaître le coefficient angulaire de cette tangente, en prenant la dérivée du second membre de l'équation . ว),

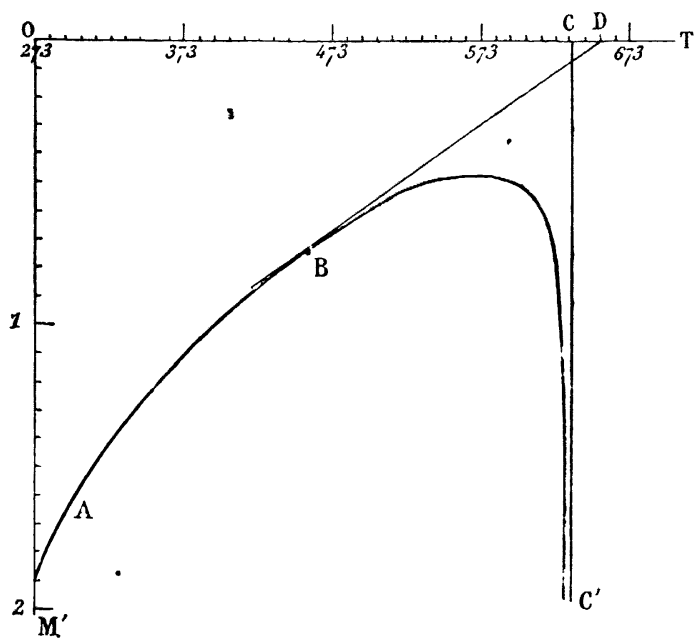

FIri. 1.

et calculer ensuite l'abscisse de son point de rencontre D avec l'axe OT. On trouve ainsi 6ăōo. Cette valeur est supérieure à celle de la température critique absolue de l'eau, qui, d'après MM. Cailletet et Colardeau, est égale à $638^{\circ}$ absolus. Soit $\mathrm{C}$ le point ayant pour abscisse cette température critique. La courbe représentant les valeurs $m^{\prime}$ est asymptote à la parallèle $\mathrm{CC}^{\prime}$ menée par le point $\mathrm{C}$ à l'axe $\mathrm{ONI}^{\prime}$; elle doit se trouver, d'ailleurs, tout entière au-dessous de la tangente $\mathrm{BD}$. Elle ne peut donc pas rencontrer l'axe des températures, et sa forme doit être voisine de celle représentée par la figure.

On est "insi conduit à regarder la rhaleur spécifique de lu

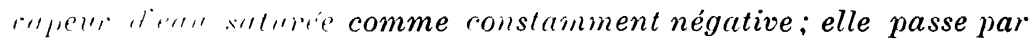
un maximum qui ne parait pas stre s"spérieur $\dot{a}-0,30$. 